\title{
Lymphatic Vessels Enhancing Adaptive Immunity Deteriorates Renal Inflammation and Renal Fibrosis
}

\author{
Jianliang Wu Guangchang Pei Rui Zeng Gang Xu \\ Division of Nephrology, Tongji Hospital, Tongji Medical College, Huazhong University of Science and Technology, \\ Wuhan, PR China
}

\author{
Keywords \\ Lymphangiogenesis · Kidney disease $\cdot$ Renal fibrosis . \\ Adaptive immunity
}

\section{Abstract}

Background: Lymphatic vessels transport lymph away from microvascular beds into the cardiovascular system. The basic function of the lymphatic system include absorption of water and macromolecules in the interstitial fluid, which plays an important role in maintaining osmotic balance of the body. Recent studies have shown that lymphangiogenesis is associated with tumor metabolism, injury repair, and chronic inflammation, and deteriorates disease progression via immune cell trafficking. Summary: Renal interstitial lymphangiogenesis is found in patients with chronic kidney disease and a series of animal models of renal fibrosis. Lymphatic vessels transfer antigen and antigen-presenting cells from peripheral tissues to lymph nodes, which initiates adaptive immunity and in turn deteriorates renal inflammation and renal fibrosis, even in non-autoimmune renal diseases. Key Messages: This review summarizes the latest findings on how lymphatics participate in the progression of chronic kidney disease. This discussion will serve to highlight the role of adaptive immunity in non-infectious and non-autoimmune nephropathy, in order to provide new ideas and methods for prevention and treatment of kidney diseases.

(c) 2020 The Author(s)

Published by S. Karger AG, Basel

\section{Introduction}

Two major circulatory systems in the human body, the cardiovascular system and the lymphatic system, were first described by Hippocrates and Aristotle in the 4th century B.C. However, it was not until the mid-17th century when Gasaro Aselli stumbled upon "lacteal vessels" when dissecting a live dog's abdomen and observed lymphatic valves that the lymphatic system research in the modern sense was developed [1]. Although lymphatics and blood vessels are similar in structure, function, and course, for a long time, due to the lack of specific markers of lymphatic endothelial cells (LECs), little has been known about the physiological functions of lymphatics and their roles in diseases. Until the discovery of unique lymphatic markers in the past two decades, the function of lymphatics has gradually attracted attention [2]. Vascular endothelial growth factor receptor 3 (VEGFR-3),

$\begin{array}{ll}\text { karger@karger.com } & \text { (c) 2020 The Author(s) } \\ \text { Pww.karger.com/kdd } & \text { Published by S. Karger AG, Basel } \\ \text { This article is licensed under the Creative Commons Attribution- } \\ \text { NonCommercial-NoDerivatives 4.0 International License (CC BY- } \\ \text { NC-ND) (http://www.karger.com/Services/OpenAccessLicense). } \\ \text { Usage and distribution for commercial purposes as well as any dis- } \\ \text { tribution of modified material requires written permission. }\end{array}$


Table 1. Summary of common lymphatic markers

\begin{tabular}{|c|c|c|}
\hline Marker & $\begin{array}{l}\text { Cellular sites } \\
\text { of expression }\end{array}$ & Molecular function \\
\hline VEGFR-3 & Cell membrane & Tyrosine kinase receptor \\
\hline Lyve-1 & Cell membrane & Hyaluronan receptor \\
\hline Prox-1 & Cell nucleus & Transcription factor \\
\hline Podoplanin & Cell membrane & Transmembrane glycoprotein \\
\hline CCL21 & & $\begin{array}{l}\text { CC-chemokine for CCR7+ } \\
\text { cells }\end{array}$ \\
\hline
\end{tabular}

also known as FLT-4, was the first relatively specific marker used to identify lymphatics [3]. Similar to VEGFR-1 and VEGFR-2, VEGFR-3 is a member of the VEGF family. As a membrane-anchored tyrosine kinase and VEGF receptor, VEGFR-3 plays a crucial role in lymphangiogenesis [4]. However, the relatively specific anchorage of VEGFR-3 on LECs occurs at the late stage of lymphatic development. Subsequently, a series of important lymphatic markers have been reported, such as lymphatic vessel endothelial hyaluronan receptor (Lyve-1), prospero-related homeobox transcription factor 1 (Prox-1), podoplanin, and so on. Lyve- 1 is an integral membrane glycoprotein important for cell migration and a new homologue of the CD44 glycoprotein $[5,6]$. Its structural characteristics suggest that lymphatic vessels (LVs) may have physiological functions of transporting hyaluronic acid. Although Gale et al. [7] reported that Lyve-1 is not compulsory for normal lymphatic development and function, we generated a Lyve-1-Cre/iDTR double-transgenic mouse in which Lyve-1-expressing LVs could be ablated by diphtheria toxin, and we found that conditional knockdown of Lyve-1-expressing LVs attenuated intrarenal inflammatory infiltration and renal fibrosis [8]. This suggests that lymphangiogenesis participates in the progression of kidney disease by regulating adaptive immunity under pathological conditions. In addition, Lyve1 was also found to be expressed on the surface of macrophages and sinusoidal cells of liver and spleen $[9,10]$. Unlike Lyve-1, Prox-1 is essential for lymphatics development as the master control gene, which encodes a nuclear transcription factor, and is sufficient to induce expression of the two well-characterized lymphatic markers podoplanin and VEGFR-3 $[11,12]$. Prox-1 $1^{-/-}$mice lack a lymphatic vascular system and die a few days after their birth [12]. Although a reliable marker of LECs, Prox- 1 can be detected in other nonendothelial cell types [13]. Mucintype transmembrane glycoprotein podoplanin is another

LVs Enhancing Adaptive Immunity

Deteriorates Renal Fibrosis gene associated with the LEC phenotype, which is expressed in LECs during the whole development period. Podoplanin was originally identified from cells of osteoblastic lineage [14] and podocytes [15]. The discovery of these lymphatic markers (Table 1) opened us the door to a comprehensive and detailed study of lymphatics in healthy and diseased tissues.

\section{LVs Serve as a Bridge between Innate and Adaptive Immunity}

The first and foremost function of the lymphatics is to maintain tissue fluid homeostasis by removing the protein-rich lymph from the extracellular space to the blood circulation and absorption of fats in the gastrointestinal tract [16]. The initial lymphatics is the entrance of almost all dietary lipids, which are absorbed by intestinal cells and packaged into large lipoproteins (chylomicrons) and exported to the LVs [17]. In most tissues under physiological conditions, when plasma enters the capillary, there is continuous leakage of plasma into extracellular space [18]. These exuded interstitial fluids and macromolecules are mostly absorbed by LVs, while intravenous reabsorption plays a small role in the majority of vascular beds [19]. Therefore, the lymphatic system is an important factor in maintaining tissue fluid balance.

While it has long been recognized that lymphatics transport immune cells, recent studies have just identified the mechanisms by which LECs directly communicate with immune cell populations and in turn regulate adaptive immunity $[20,21]$. Lymphatics also regulate innate immunity through their roles in uptake and transport of soluble antigens and ability to regulate lymphatic flow. Therefore, LVs serve as a bridge between innate and adaptive immunity, playing an important role in various physiological and pathological conditions. Immune cells found in lumen of LVs include T and B lymphocytes, monocytes, macrophages, dendritic cells (DCs), neutrophils, and eosinophils. Red blood cells and activated plasma cells secreting antibodies can be detected in the course of immune response. Tumor cells were identified entering initial lymphatics by using chemokine gradient guidance via open junction, leading to distant metastasis of primary tumors. The LVs are essential for trafficking of immune cells and soluble antigens from peripheral tissues to draining lymph nodes [22]. The basic function of lymph is to remove pathogens that invade tissues. The composition of cells in lymph is also dependent upon the presence of antigens and inflammation. In fact, by drain- 
ing through the lymph nodes, the lymphatic system ensures tissue-invading pathogens captured by macrophages and DCs in LVs and prohibits their flowing directly into the bloodstream, causing serious consequences [21, 23]. However, immune cells not only migrate through LVs, but also interact with LVs and promote lymphangiogenesis $[21,24]$. A large amount of data support a close interaction between inflammation and lymphangiogenesis. At the site of inflammation, lymphatics transport immune cells, cytokines, and chemokines to lymph nodes, while certain cytokines secreted by immune cells promote lymphangiogenesis. Therefore, LVs are not only a passive participant but also an active promoter in many pathophysiological processes.

DCs are the most effective antigen-presenting cells in the body, which are essential for presenting peripheral antigens and initiating adaptive immune responses [25]. Under physiological conditions, relatively few but stable DCs enter the afferent lymphatics. This steady entry seems to play a role in maintaining immune tolerance to autoantigens [26]. In contrast, after the onset of inflammation, lymphangiogenesis occurs in the tissue and more activated mature DCs enter the peripheral tissue. This, together with an increased expression of CCL21 and other chemokine and integrin pathways, drives increased transit of DCs to the lymphatic system [25]. This process involves the degradation of pathogenic proteins into presentable antigens and upregulates the expression of CCR7 and other co-receptors for T-cell activation [27]. The migration of DCs to LVs requires the involvement of multiple chemokines and their receptors, especially CCR7/ CCL2 1 axis, in both the steady state and during inflammation $[26,28]$. CCL19 is also secreted by LECs, but in very small amounts compared with CCL21 [29]. In addition, inflammatory mediators such as TNF- $\alpha$ and IL- $1 \beta$ also stimulate production of chemokines and chemokine receptors to mobilize and activate DCs [30]. Upon entry into lymphatics, DCs ferry their soluble antigen toward the lymph nodes [31].

Memory T cells also enter initial lymphatics [25]. Tissue-resident memory cells lack CCR7, but migrating memory $\mathrm{T}$ cells express CCR7 and have the capacity to enter initial LVs, following the CCL21 gradient guidance [32]. T cell entry into afferent lymphatics is regulated by the bioactive lipid sphingosine-1-phosphate (S1P), which activates specific S1P receptors on the surface of T lymphocytes to direct migration [33]. There are less B lymphocytes presenting in both afferent and efferent lymph.

An increase in number of LVs helps interstitial immune cells draining away from the site of inflammation and accelerates the resolution of inflammation [24, 34, 35]. However, LVs, as a channel for immune cell transportation, play different roles in different organs and pathological conditions $[34,35]$. We demonstrate that lymphangiogenesis in kidneys and renal draining lymph nodes deteriorates unilateral ureteral obstruction (UUO)induced intrarenal inflammation and the consequent fibrosis, which was attenuated by conditional knockdown of intrarenal LVs [8].

Both innate and adaptive immunity play an important role in the development of chronic kidney disease [36]. Apoptosis and necrosis after renal injury act as endogenous DAMP to irritate DCs, macrophages, vascular endothelial cells, mesangial cells, podocytes, and tubular epithelial cells to secrete a series of inflammatory mediators, which promotes the progress of the disease in the innate immune phase. For example, macrophages consist of two subtypes, the pro-inflammatory M1 type and an anti-inflammatory M2 type. It has been reported that M1 type macrophages promote renal fibrosis requiring the presence of COX-2 [37]. Our research team sorted out Ly6C-macrophages in mice kidney at 5 days after IR operation and injected them under the renal capsule of mice with immune deficiency, finding that the original normal kidney showed damage and intrarenal fibrosis 5 days later, suggesting that Ly6C- macrophages directly damaged kidney and induced subsequent fibrosis [38]. We further identified that Ly6C- macrophages were the dominant intrarenal macrophages after ischemia-reperfusion injury, especially at the chronic phase, and most were derived from the bone marrow and depended on intrarenal CX3CL1-CX3CR1 interaction [38].

The role of adaptive immunity in kidney disease cannot be ignored either. Under physiological conditions, a small number of DCs enter the LVs through the CCR7CCL19/21 signaling pathway and then are drained to the corresponding lymph nodes. If DCs are expanded by the stimulation of the antigen and entering the LVs to the draining lymph nodes, this will initiate acquired immunity and aggravate the kidney damage in the site of inflammation [25]. Intestinal flora-derived Th17 cells enter the LVs through the S1P-R1 pathway to the peripheral blood circulation and then reach the renal inflammation site deteriorating renal inflammation. On the contrary, the application of broad-spectrum combined antibiotics to inhibit intestinal flora and the following Th17 cell activation protects autoimmune kidney disease, suggesting that intestinal-derived Th17 cells can move to kidney and induce kidney disease via LV system communication [39]. Tapmeier et al. [40] found a critical role for CD4(+) 
T cells in kidney fibrosis using lymphopenic RAG(-/-) mice after ureteric obstruction. In addition, early-stage accumulation of B cells in the kidney accelerated monocyte/macrophage mobilization and infiltration and aggravated the renal fibrosis [41].

\section{Lymphangiogenesis and Kidney Disease}

Early studies of lymphatic-related diseases focused on lymphedema caused by lymphatic reflux disorders. LVs have been found to play a pivotal role in immune-related diseases in the recent two decades. A large amount of literature reports that lymphangiogenesis is often associated with pathological conditions, such as tumor metastasis [42], injury repair [43], and chronic inflammation [44]. Renal lymphangiogenesis is associated with renal fibrosis, inflammation, and transplant rejection. In healthy renal tissue, only a small number of lymphatic capillaries are distributed around the interlobular artery and interlobular vein, without distribution in the glomerulus and renal interstitium [45]. However, in chronic kidney diseases such as lupus nephritis, anti-neutrophil cytoplasmic antibody-related glomerulonephritis, tubulointerstitial nephritis, focal segmental glomerulosclerosis, crescentic glomerulonephritis, type II diabetic nephropathy, and IgA nephropathy, it shows markedly increased numbers of LVs compared to controls $[8,46]$. Numerous studies have shown that proteinuria induces the expression and release of chemokines and mediators in renal tubular epithelial cells, leading to inflammatory cell recruitment and kidney damage [46], which triggers intrarenal lymphangiogenesis before the onset of interstitial fibrosis. In a rat proteinuric model, lymphangiogenesis occurred prior to macrophage influx, collagen deposition, and interstitial fibrosis, suggesting a possible pathogenetic role in fibrosis [45].

Various growth factors and mediators have been shown to promote lymphangiogenesis in different organs [47]. Vascular endothelial growth factor (VEGF)-C and VEGF-D are most widely known to promote lymphangiogenesis. VEGF-C binds to VEGFR-2 and VEGFR-3, which supports the survival, proliferation, and migration of LECs [48]. Hasegawa et al. [48] found that lymphangiogenesis ameliorates inflammation and fibrosis via the VEGF-C signaling pathway. In addition, VEGF-D is another major promoter of lymphangiogenesis, also depending on VEGFR-3, but its ability to promote lymphangiogenesis is less important than that of VEGF-C. The injury tubules produce a large amount of VEGF-C and

LVs Enhancing Adaptive Immunity

Deteriorates Renal Fibrosis
VEGF-D during acute kidney injury and chronic kidney disease [49]. Kinashi et al. [50] found that connective tissue growth factor (CTGF) is another important determinant of lymphangiogenesis. The increase of LVs and VEGF-C in obstructed and ischemia-reperfusion injury kidneys was significantly reduced in CTGF knockout compared to wild-type mice. Similarly, TGF- $\beta 1$ upregulated the expression of VEGF-C in tubular epithelial cells, and TGF- $\beta 1$ receptor inhibitor LY364947 inhibited the upregulation of VEGF-C and lymphangiogenesis in the kidneys [51]. These data confirmed that damaged renal tubules not only act as victims but also as an active participant of the progression of kidney disease, which promotes lymphangiogenesis and the development of chronic kidney disease by producing TGF- $\beta 1$, CTGF, and other cytokines.

Chronic kidney disease is a chronic inflammatory disease, associated with migration and activation of leukocytes and an accumulation of excess interstitial fluid. Theoretically, lymphangiogenesis occurs at the inflamed site to clear antigen fluid, cytokines, and macromolecules. However, whether LVs serve to resolve or exacerbate inflammation remains a matter of debate. A recent study indicates that VEGF-C induced expression of VEGFR-3 and lymphangiogenesis in the interstitium and suppressed interstitial fibrosis in UUO kidneys. VEGF-C also reduced collagen 1 and TGF- $\beta 1$ expression as well as macrophage accumulation in the interstitium [48]. The protective effect of lymphangiogenesis is also reported in inflammatory bowel disease [52] and diabetic wounds [53]. However, our data in kidney showed that blocking recruitment of CCR7+ cells into renal draining lymph nodes and the spleen through inhibition of lymphangiogenesis or administration of a CCR7-neutralizing antibody markedly attenuated intrarenal inflammation and fibrosis. Continuous and irreversible injury inflicted by UUO or ischemia-reperfusion injury led to lymphangiogenesis with upregulated CCL21 expression and recruitment of CCR7+ immune cells into the renal draining lymph nodes and spleen. This ultimately led to exacerbation of renal inflammation and fibrosis, rather than inflammation resolution [8]. A lot of recent literature also supports our view $[54,55]$.

Therefore, we assumed that after kidney injury, the intrinsic lymphatic drainage function of kidney is unobstructed in the early stage of renal inflammation injury. A small number of CCR7+ immune cells with antigen-presenting potential are transported through the LVs of CCL21+ to the lymph nodes around the kidney, resulting in the activation and proliferation of lymphocytes, which 
Fig. 1. Hypothesis of the effects of lymphangiogenesis on "splenic-renal crosstalk."

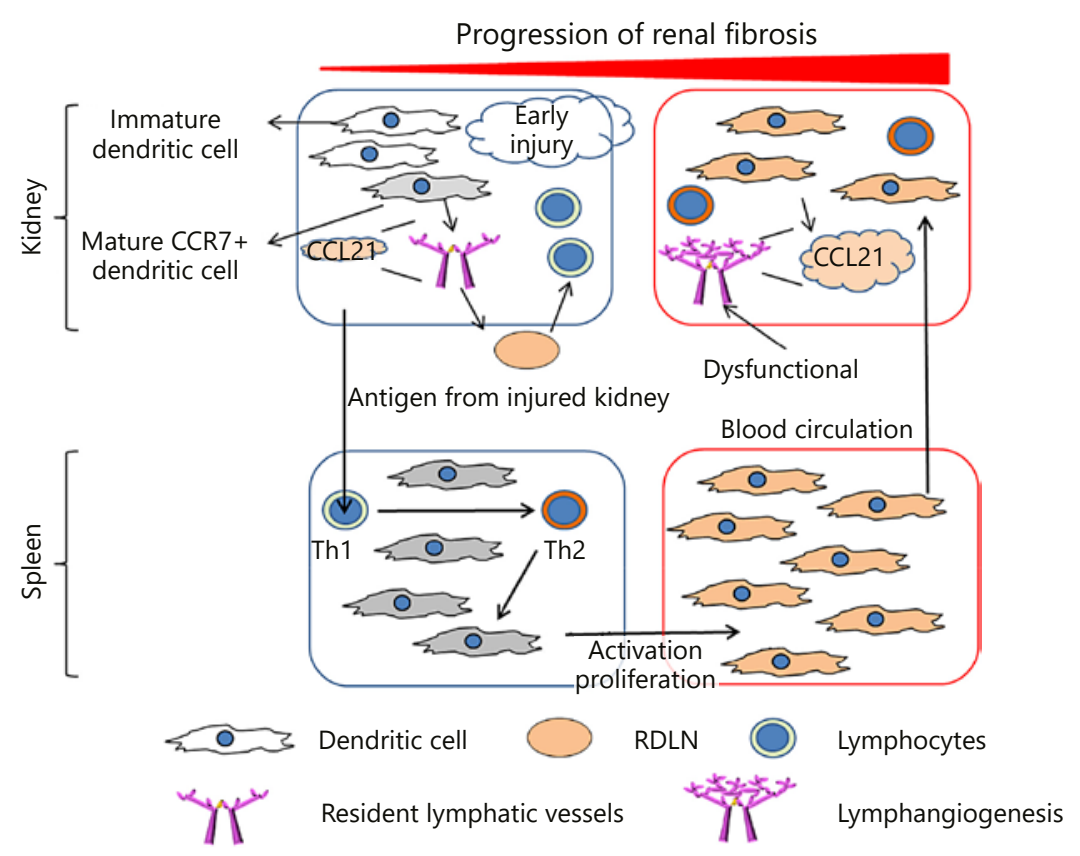

participate in the inflammatory damage of kidney. The renal injury signal is transported to the spleen through the blood circulation, causing the activation phenotype of spleen lymphocytes to change, the proportion of Th1 cells decreases, and the proportion of Th2 cells increases, which leads to the proliferation and activation of spleen CCR7+ lymphocytes. Activated spleen DC, T-cell antigen presentation, and secretion of chemokines are significantly enhanced and released into the blood circulation in large quantities. At the same time, the inflammatory factors and growth factors derived from the microenvironment of kidney damage lead to the extension and expansion of the intrinsic LVs. LECs activate and secrete a large amount of CCL21 under the action of factors such as kidney-derived TNF- $\alpha$, leading to chronic inflammation and promoting the progression of renal fibrosis (Fig. 1).

\section{Prospect for the Future}

As mentioned above, both blocking and promoting lymphangiogenesis have been suggested to be advantageous or disadvantageous, implying to be highly context dependent and organ specific. The newborn LVs serve as an excessive inflammatory cell output channel, thereby attenuating chronic inflammatory response. Inducing lymphangiogenesis has been shown to decrease intersti- tial fluid accumulation and attenuate inflammation [56]. Muchowicz et al. [54] reported that sustained inhibition of lymphangiogenesis by soluble VEGFR-3 also inhibited the capacity of DCs translocating into local lymph nodes and resulted in less tumor antigen-specific CD8+ T-cell response. Lymphangiogenesis is also associated with chronic kidney allograft injury, and sirolimus has been identified as a potent inhibitor of lymphangiogenesis in renal allografts by blocking the VEGF-C/VEGFR-3 pathway [57]. We also found that blocking CCR7+ cell recruitment into LVs via genetic and pharmacologic approaches or using CCR7 neutralizing antibody attenuated intrarenal inflammation and fibrosis [8]. Although there is still much debate on the function and mechanism of lymphatics, our data support the view that lymphatics enhancing adaptive immunity deteriorate renal inflammation and renal fibrosis in chronic kidney disease. These data suggest that targeting on lymphangiogenesis in kidney and renal draining lymph nodes is a new strategy for prevention and treatment of kidney diseases. We hope to find specific targets related to lymphatics in the future to delay the progress of chronic kidney disease.

\section{Disclosure Statement}

The authors have no conflicts of interest to declare. 


\section{Funding Sources}

This work was supported by the Major Research Plan of the National Natural Science Foundation of China (grant No. 91742204), International (regional) Cooperation and Exchange Projects (NSFC-DFG, grant No. 81761138041), the National Natural Science Foundation of China for Young Scholars (grant No. 81600556).

\section{Author Contributions}

J.W. and G.P. wrote the paper. R.Z. and G.X. conceived the project and supervised and coordinated all the work. All authors have read and approved the final manuscript.

\section{References}

1 Louveau A, Smirnov I, Keyes TJ, Eccles JD, Rouhani SJ, Peske JD, et al. Structural and functional features of central nervous system lymphatic vessels. Nature. 2015 Jul;523(7560): 337-41.

2 Choi I, Lee S, Hong YK. The new era of the lymphatic system: no longer secondary to the blood vascular system. Cold Spring Harb Perspect Med. 2012 Apr;2(4):a006445.

3 Kaipainen A, Korhonen J, Mustonen T, van Hinsbergh VW, Fang GH, Dumont D, et al. Expression of the fms-like tyrosine kinase 4 gene becomes restricted to lymphatic endothelium during development. Proc Natl Acad Sci USA. 1995 Apr;92(8):3566-70.

4 Tacconi C, Ungaro F, Correale C, Arena V, Massimino L, Detmar M, et al. Activation of the VEGFC/VEGFR3 Pathway Induces Tumor Immune Escape in Colorectal Cancer. Cancer Res. 2019 Aug;79(16):4196-210.

5 Prevo R, Banerji S, Ferguson DJ, Clasper S, Jackson DG. Mouse LYVE-1 is an endocytic receptor for hyaluronan in lymphatic endothelium. J Biol Chem. 2001 Jun;276(22): 19420-30.

6 Banerji S, Ni J, Wang SX, Clasper S, Su J, Tam$\mathrm{mi}$, et al. LYVE-1, a new homologue of the CD44 glycoprotein, is a lymph-specific receptor for hyaluronan. J Cell Biol. 1999 Feb; 144(4):789-801.

7 Gale NW, Prevo R, Espinosa J, Ferguson DJ, Dominguez MG, Yancopoulos GD, et al. Normal lymphatic development and function in mice deficient for the lymphatic hyaluronan receptor LYVE-1. Mol Cell Biol. 2007 Jan; 27(2):595-604.

8 Pei G, Yao Y, Yang Q, Wang M, Wang Y, $\mathrm{Wu}$ J, et al. Lymphangiogenesis in kidney and lymph node mediates renal inflammation and fibrosis. Sci Adv. 2019 Jun; 5(6):eaaw5075.

9 Mouta Carreira C, Nasser SM, di Tomaso E, Padera TP, Boucher Y, Tomarev SI, et al. LYVE-1 is not restricted to the lymph vessels: expression in normal liver blood sinusoids and down-regulation in human liver cancer and cirrhosis. Cancer Res. 2001 Nov;61(22): 8079-84.

10 Arimoto J, Ikura Y, Suekane T, Nakagawa M, Kitabayashi C, Iwasa Y, et al. Expression of LYVE-1 in sinusoidal endothelium is reduced in chronically inflamed human livers. J Gastroenterol. 2010 Mar;45(3):317-25.
11 Wigle JT, Harvey N, Detmar M, Lagutina I, Grosveld G, Gunn MD, et al. An essential role for Proxl in the induction of the lymphatic endothelial cell phenotype. EMBO J. 2002 Apr;21(7):1505-13.

12 Hong YK, Detmar M. Prox1, master regulator of the lymphatic vasculature phenotype. Cell Tissue Res. 2003 Oct;314(1):85-92.

13 Truman LA, Bentley KL, Smith EC, Massaro SA, Gonzalez DG, Haberman AM, et al. ProxTom lymphatic vessel reporter mice reveal Prox1 expression in the adrenal medulla, megakaryocytes, and platelets. Am J Pathol. 2012 Apr;180(4):1715-25.

14 Wetterwald A, Hoffstetter W, Cecchini MG, Lanske B, Wagner C, Fleisch H, et al. Characterization and cloning of the E11 antigen, a marker expressed by rat osteoblasts and osteocytes. Bone. 1996 Feb;18(2):125-32.

15 Breiteneder-Geleff S, Matsui K, Soleiman A, Meraner P, Poczewski H, Kalt R, et al. Podoplanin, novel $43-\mathrm{kd}$ membrane protein of glomerular epithelial cells, is down-regulated in puromycin nephrosis. Am J Pathol. 1997 Oct; 151(4):1141-52.

16 Breslin JW, Yang Y, Scallan JP, Sweat RS, Adderley SP, Murfee WL. Lymphatic Vessel Network Structure and Physiology. Compr Physiol. 2018 Dec;9(1):207-99.

17 Hokkanen K, Tirronen A, Ylä-Herttuala S. Intestinal lymphatic vessels and their role in chylomicron absorption and lipid homeostasis. Curr Opin Lipidol. 2019 Oct;30(5): 370-6.

18 Renkin EM. Some consequences of capillary permeability to macromolecules: starling's hypothesis reconsidered. Am J Physiol. 1986 May;250(5 Pt 2):H706-10.

19 Levick JR, Michel CC. Microvascular fluid exchange and the revised Starling principle. Cardiovasc Res. 2010 Jul;87(2):198-210.

20 Yeo KP, Angeli V. Bidirectional Crosstalk between Lymphatic Endothelial Cell and T Cell and Its Implications in Tumor Immunity. Front Immunol. $2017 \mathrm{Feb} ; 8: 83$.

21 Betterman KL, Harvey NL. The lymphatic vasculature: development and role in shaping immunity. Immunol Rev. 2016 May;271(1): 276-92.

22 Jiang X, Nicolls MR, Tian W, Rockson SG Lymphatic Dysfunction, Leukotrienes, and Lymphedema. Annu Rev Physiol. 2018 Feb; 80(1):49-70.
23 Randolph GJ, Ivanov S, Zinselmeyer BH, Scallan JP. The Lymphatic System: Integral Roles in Immunity. Annu Rev Immunol. 2017 Apr;35(1):31-52.

24 Kataru RP, Lee YG, Koh GY. Interactions of immune cells and lymphatic vessels. Adv Anat Embryol Cell Biol. 2014;214:107-18.

25 Platt AM, Randolph GJ. Dendritic cell migration through the lymphatic vasculature to lymph nodes. Adv Immunol. 2013;120:5168.

26 Ohl L, Mohaupt M, Czeloth N, Hintzen G, Kiafard Z, Zwirner J, et al. CCR7 governs skin dendritic cell migration under inflammatory and steady-state conditions. Immunity. 2004 Aug;21(2):279-88.

27 MartIn-Fontecha A, Sebastiani S, Höpken UE, Uguccioni M, Lipp M, Lanzavecchia A, et al. Regulation of dendritic cell migration to the draining lymph node: impact on T lymphocyte traffic and priming. J Exp Med. 2003 Aug;198(4):615-21.

28 Johnson LA, Jackson DG. Inflammation-induced secretion of CCL21 in lymphatic endothelium is a key regulator of integrin-mediated dendritic cell transmigration. Int Immunol. 2010 Oct;22(10):839-49.

29 Britschgi MR, Favre S, Luther SA. CCL21 is sufficient to mediate DC migration, maturation and function in the absence of CCL19. Eur J Immunol. 2010 May;40(5):1266-71.

30 Enk AH, Angeloni VL, Udey MC, Katz SI. An essential role for Langerhans cell-derived IL-1 beta in the initiation of primary immune responses in skin. J Immunol. 1993 May;150(9): 3698-704.

31 Johnson LA, Banerji S, Lawrance W, Gileadi U, Prota G, Holder KA, et al. Dendritic cells enter lymph vessels by hyaluronan-mediated docking to the endothelial receptor LYVE-1. Nat Immunol. 2017 Jul;18(7):762-70.

32 Hampton HR, Chtanova T. Lymphatic Migration of Immune Cells. Front Immunol. 2019 May; 10:1168.

33 Maeda Y, Seki N, Sato N, Sugahara K, Chiba K. Sphingosine 1-phosphate receptor type 1 regulates egress of mature $\mathrm{T}$ cells from mouse bone marrow. Int Immunol. 2010 Jun;22(6): $515-25$.

34 Abouelkheir GR, Upchurch BD, Rutkowski JM. Lymphangiogenesis: fuel, smoke, or extinguisher of inflammation's fire? Exp Biol Med (Maywood). 2017 Apr;242(8):884-95. 
35 Kim H, Kataru RP, Koh GY. Inflammationassociated lymphangiogenesis: a doubleedged sword? J Clin Invest. 2014 Mar;124(3): 936-42.

36 Kurts C, Panzer U, Anders HJ, Rees AJ. The immune system and kidney disease: basic concepts and clinical implications. Nat Rev Immunol. 2013 Oct;13(10):738-53.

37 Wang X, Yao B, Wang Y, Fan X, Wang S, Niu A, et al. Macrophage Cyclooxygenase-2 Protects Against Development of Diabetic Nephropathy. Diabetes. 2017 Feb;66(2):494504.

38 Yang Q, Wang $\mathrm{Y}$, Pei G, Deng $\mathrm{X}$, Jiang $\mathrm{H}$, Wu $\mathrm{J}$, et al. Bone marrow-derived Ly6C- macrophages promote ischemia-induced chronic kidney disease. Cell Death Dis. 2019 Mar; 10(4):291.

39 Krebs CF, Paust HJ, Krohn S, Koyro T, Brix SR, Riedel JH, et al. Autoimmune Renal Disease Is Exacerbated by S1P-Receptor-1-Dependent Intestinal Th17 Cell Migration to the Kidney. Immunity. 2016 Nov;45(5):1078-92.

40 Tapmeier TT, Fearn A, Brown K, Chowdhury P, Sacks SH, Sheerin NS, et al. Pivotal role of $\mathrm{CD} 4+\mathrm{T}$ cells in renal fibrosis following ureteric obstruction. Kidney Int. 2010 Aug;78(4): 351-62.

41 Han H, Zhu J, Wang Y, Zhu Z, Chen Y, Lu L, et al. Renal recruitment of $B$ lymphocytes exacerbates tubulointerstitial fibrosis by promoting monocyte mobilization and infiltration after unilateral ureteral obstruction. J Pathol. 2017 Jan;241(1):80-90.

42 Xiong Y, Liu Z, Zhao X, Ruan S, Zhang X, Wang $S$, et al. CPT1A regulates breast cancerassociated lymphangiogenesis via VEGF signaling. Biomed Pharmacother. 2018 Oct;106: $1-7$.
43 Güç E, Briquez PS, Foretay D, Fankhauser MA, Hubbell JA, Kilarski WW, et al. Local induction of lymphangiogenesis with engineered fibrin-binding VEGF-C promotes wound healing by increasing immune cell trafficking and matrix remodeling. Biomaterials. 2017 Jul;131:160-75.

44 Edwards LA, Nowocin AK, Jafari NV, Meader LL, Brown K, Sarde A, et al. Chronic Rejection of Cardiac Allografts Is Associated With Increased Lymphatic Flow and Cellular Trafficking. Circulation. 2018 Jan;137(5):488503.

45 Yazdani S, Navis G, Hillebrands JL, van Goor $\mathrm{H}$, van den Born J. Lymphangiogenesis in renal diseases: passive bystander or active participant? Expert Rev Mol Med. 2014 Sep; 16:e15.

46 Zimmer JK, Dahdal S, Mühlfeld C, Bergmann IP, Gugger M, Huynh-Do U. Lymphangiogenesis is upregulated in kidneys of patients with multiple myeloma. Anat Rec (Hoboken). 2010 Sep;293(9):1497-505.

47 Zheng W, Aspelund A, Alitalo K. Lymphangiogenic factors, mechanisms, and applications. J Clin Invest. 2014 Mar;124(3):878-87.

48 Hasegawa S, Nakano T, Torisu K, Tsuchimoto A, Eriguchi M, Haruyama N, et al. Vascular endothelial growth factor-C ameliorates renal interstitial fibrosis through lymphangiogenesis in mouse unilateral ureteral obstruction. Lab Invest. 2017 Dec;97(12):1439-52.

49 Zarjou A, Black LM, Bolisetty S, Traylor AM, Bowhay SA, Zhang MZ, et al. Dynamic signature of lymphangiogenesis during acute kidney injury and chronic kidney disease. Lab Invest. 2019 Sep;99(9):1376-88.
50 Kinashi H, Falke LL, Nguyen TQ, Bovenschen $\mathrm{N}$, Aten J, Leask A, et al. Connective tissue growth factor regulates fibrosis-associated renal lymphangiogenesis. Kidney Int. 2017 Oct; 92(4):850-63.

51 Suzuki Y, Ito Y, Mizuno M, Kinashi H, Sawai A, Noda $Y$, et al. Transforming growth factor- $\beta$ induces vascular endothelial growth factor- $\mathrm{C}$ expression leading to lymphangiogenesis in rat unilateral ureteral obstruction. Kidney Int. 2012 May;81(9):865-79.

52 D'Alessio S, Correale C, Tacconi C, Gandelli A Pietrogrande G, Vetrano S, et al. VEGF-C-dependent stimulation of lymphatic function ameliorates experimental inflammatory bowel disease. J Clin Invest. 2014 Sep;124(9):3863-78.

53 Saaristo A, Tammela T, Farkkilā A, Kärkkäinen M, Suominen E, Yla-Herttuala S, et al. Vascular endothelial growth factor-C accelerates diabetic wound healing. Am J Pathol. 2006 Sep;169(3):1080-7.

54 Muchowicz A, Wachowska M, Stachura J, Tonecka K, Gabrysiak M, Wołosz D, et al. Inhibition of lymphangiogenesis impairs antitumour effects of photodynamic therapy and checkpoint inhibitors in mice. Eur J Cancer. 2017 Sep;83:19-27.

55 Lund AW, Wagner M, Fankhauser M, Steinskog ES, Broggi MA, Spranger S, et al. Lymphatic vessels regulate immune microenvironments in human and murine melanoma. J Clin Invest. 2016 Sep;126(9):3389-402.

56 Kim H, Kataru RP, Koh GY. Regulation and implications of inflammatory lymphangiogenesis. Trends Immunol. 2012 Jul;33(7):350-6.

57 Palin NK, Savikko J, Koskinen PK. Sirolimus inhibits lymphangiogenesis in rat renal allografts, a novel mechanism to prevent chronic kidney allograft injury. Transpl Int. 2013 Feb;26(2):195-205 